\title{
Strengthening of Concrete, Metallic and Timber Construction Materials with FRP Composites
}

\author{
S.T. Smith (stsmith@,hku.hk) \\ Department of Civil Engineering, The University of Hong Kong, China
}

\begin{abstract}
This paper provides a review of research being conducted at The University of Hong Kong (HKU) on the strengthening of concrete, metallic and timber construction materials with externally bonded fibre-reinforced polymer (FRP) composites. The motivation of such research is to enhance our understanding of the interfacial behaviour between the FRP and the three different substrate materials via FRP-to-concrete, metal, and-timber joint tests. Of the three substrate materials, concrete has clearly experienced the most research activity to date by the wider research community, followed by that of metal and then that of timber. In order to progress the extensive knowledge base of FRP-to-concrete behaviour, research at HKU is aimed at enhancing the bond strength of the FRP via the addition of anchorage. The anchor of choice is the so called FRP anchor and its effectiveness in anchoring FRP flexural strengthening applied to reinforced concrete (RC) slabs is also presented. For the metal and timber substrate materials, HKU research is more focused at this stage on understanding the influence of key fundamental variables affecting interfacial behaviour such as FRP geometry, surface preparation and substrate material characteristics.
\end{abstract}

\section{INTRODUCTION}

Reinforced concrete (RC), metal and timber are three very commonly used construction materials. Structures made from these materials may, however, be in need of strengthening or repair or even restoration. Externally bonded fibre-reinforced polymer (FRP) composites offer a viable solution (Hollaway and Teng 2008). Considerable research to date has been conducted on the application of FRP to RC structural elements and such research has shown the propensity of the FRP to debond at strains well below its strain capacity. Our understanding of the debonding phenomenon has been enhanced via FRPto-concrete joint testing. The same experimental approach has been extended to a lesser extent to FRPto-metal interfaces. Application of FRP to timber is comparatively less again.

Research of an experimental, analytical and numerical nature is being conducted at The University of Hong Kong (HKU) which is aimed at enhancing our understanding of the application of FRP to the three very different materials of concrete, metal and timber. Such research is at various stages of development and an overview of selected experimental research to date is provided in this paper. For the case of FRP-to-concrete interfaces, enhancement of the strength of the bond between the FRP and concrete via anchorage with so called FRP anchors is being explored. The proof of the anchorage concept is also demonstrated in tests on FRP-strengthened RC slabs anchored with FRP anchors. Research on the strength and behaviour of FRP-to-metal and FRP-to-timber joints is also presented. Fundamental variables affecting interfacial behaviour, such as surface preparation and material type for metal joints, as well as natural growth characteristics in the timber for timber joints, are being investigated. Comments regarding suitable analytical modeling approaches are also made in light of the experimental findings obtained to date. Overall comments about the three different materials are also provided.

\section{FRP-TO-CONCRETE}

It has been well established that FRP plates bonded to concrete surfaces can debond at strains well below the strain capacity of the FRP (Hollaway and Teng 2008). The strain capacity of the FRP can be increased by suppression, or at least delay, of debonding by the addition of anchorage. The anchor of choice by the HKU group is the so called FRP anchor (also known as an FRP spike anchor). An FRP anchor, which is a collection of rolled fibre sheets or bundled fibre strands, is shown in Figure 1a. This figure also shows how the FRP anchor is used to anchor an externally bonded FRP plate. 


\subsection{FRP-to-concrete joint tests}

Tests on single-shear FRP-to-concrete joints, anchored with FRP anchors (Figure 1b, 2a), have proven the effectiveness of FRP anchors. The addition of a single anchor has been shown to increase the strength and slip capacity above that of an unanchored joint by $70 \%$ and $800 \%$, respectively. A recent review of research undertaken by the HKU group, as well as other groups, was presented in Smith (2009). Such research on single anchored joints by the HKU group addressed parameters of (i) fibre type (carbon and glass fibres), (ii) method of anchor formation (dry anchor or impregnated anchor), (iii) anchor position, and (iv) installation. In all cases, the anchor consisted of a single anchor fan. As shown in Figure 2a, the fan was oriented in the direction of applied load. More recent studies on single fan FRP anchors have addressed the influence of multiple FRP anchors on FRP-to-concrete joints (Zhang et al. 2010).

The encouraging performance of single fan FRP anchors has paved the way for the development of double fan FRP anchors. Such double fanned anchors, as shown in Figures 1a and 2b, are herein referred to as bow-tie FRP anchors. (a) FRP anchor and FRP plate

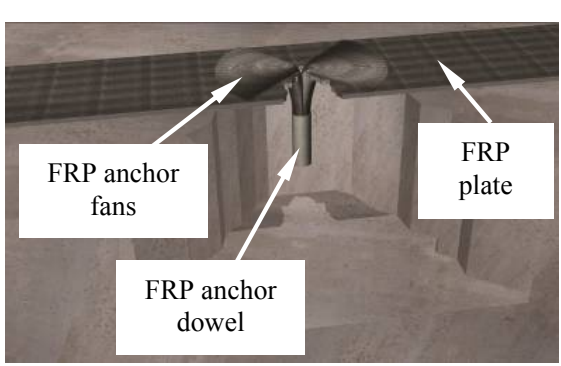

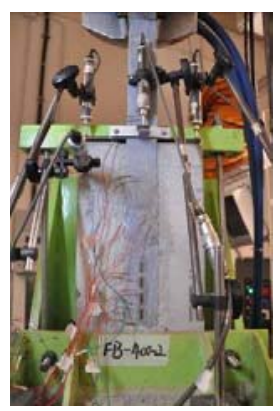

(b) Joint test set-up
Figure 1. (a) FRP plate anchored with an FRP anchor.

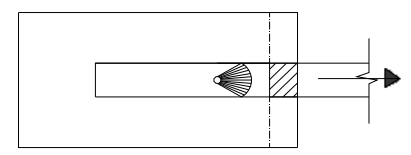

(a) Single fan anchor

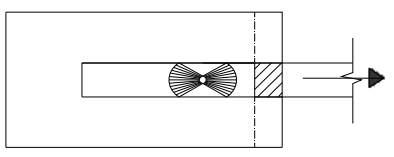

(b) Bow-tie anchor
Figure 2. FRP-to-concrete joints anchored with two different types of FRP Anchors.

Figure 3 shows the typical load-slip responses of two nominally identical FRP-to-concrete joints. The joints, which were tested in the set-up shown in Figure $1 \mathrm{~b}$, were anchored with a bow-tie FRP anchor or no anchor at all. The enhancement in load and slip capacity of the anchored joint is most evident and the three main stages in its load-slip response are herein described. It is important to note that the single and bow-tie anchors behaved in a similar manner for stages $\mathrm{A}$ and $\mathrm{B}$. For stage $\mathrm{C}$, the behaviour differed.
Stage A: Debonding initiated at the loaded end of the joint at approximately the same load to initiate debonding in an unanchored joint. The anchor was then engaged once the debonding crack passed by it. At this stage, the enhancement in joint strength above that of an unanchored joint was due to the engaged anchor and the remaining bonded FRP plate. The joint stiffness reduced as the debonding crack increased in length.

Stage B: The large drop of load at the beginning of this stage was associated with complete debonding of the FRP plate. The load then increased from friction due to sliding between the roughened surfaces of the debonded plate and concrete. In addition, the flexible FRP anchor enabled the debonded FRP plate to remain clamped to the concrete.

Stage C: The load reduced at the beginning of this stage due to partial rupture of the anchor dowel closest to the direction of applied load. A relatively constant load was then maintained from friction and the clamping effect of the remaining intact anchor. The anchor eventually completely ruptured after the slip exceeded over 10 times the slip capacity of an unanchored joint. This stage of behaviour does not occur in joints anchored with single anchor fans due to complete failure of the anchor at the end of stage B.

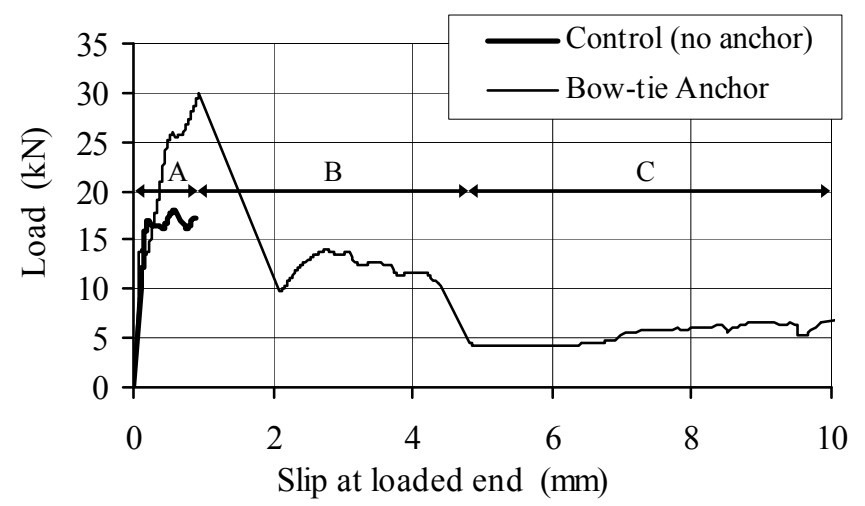

Figure 3. Load-slip responses.

\subsection{Anchored FRP-strengthened RC slab tests}

The effectiveness of the bow-tie FRP anchor in anchoring FRP flexurally-strengthened RC slabs is described in Smith et al.'s (2010) study. A summary of some of the results is presented herein.

Figure 4 is a schematic of Smith et al.'s (2010) slab specimens and test set-up. Figure 5a shows the resulting load-midspan deflection responses for three of the slabs, namely (i) unstrengthened and unanchored control slab (Slab S1), (ii) strengthened but unanchored control slab (Slab S2), and (iii) strengthened and anchored control slab (Slab S5). The FRP plate and bow-tie FRP anchor layout for Slab S5 is also shown in Figure 5b. The load-deflection response for Slab S5 reveals the anchorage to enhance 
the load carrying capacity and deflection capacity by $24 \%$ and $64 \%$, respectively, above that of Slab S2 (note: these increases are relative to the peak load the following large drop in load represents complete debonding of the FRP plate). The optimal layout of anchors produced strength and deflection gains of up to $30 \%$ and $110 \%$, respectively, however such results are not presented in this paper. The post-peak response of Slab S5 represents residual strength offered by sliding friction between the debonded FRP and concrete substrate and clamping from the anchorage. The response of Slab S5 in Figure 5a also demonstrates significant energy absorption capacity. The extent of debonding crack propagation and the conditions of the anchors at complete plate debonding is shown in Figure 5b.

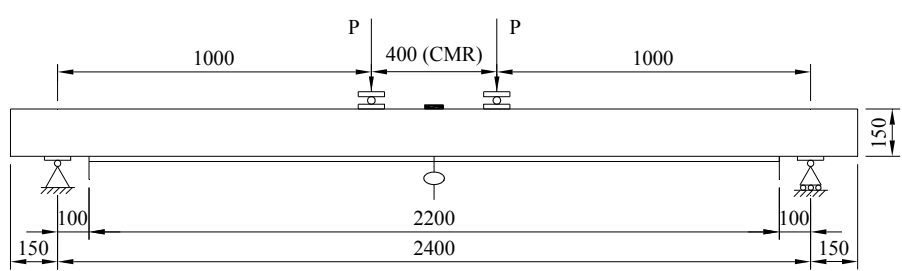

(a) Elevation

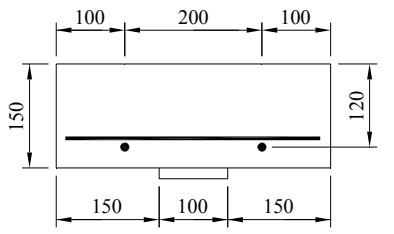

(b) Section

Figure 4. FRP-anchored FRP-strengthened slab test details.

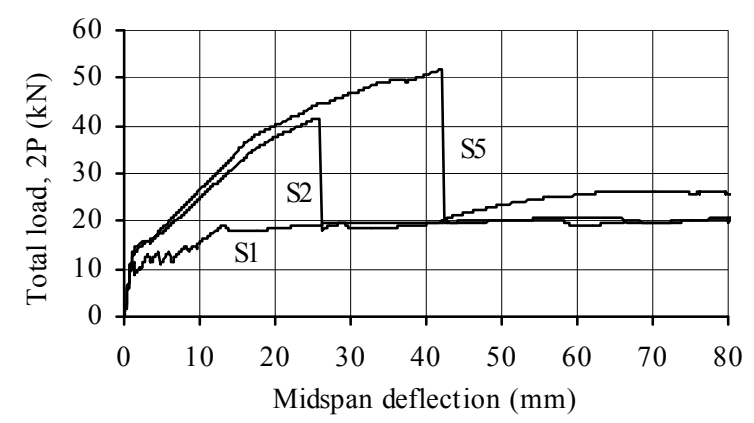

(a) Load-deflection responses

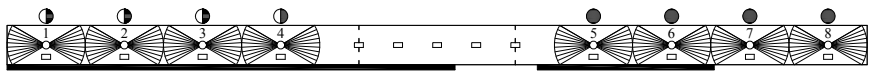

(b) Anchor layout + debonding crack extent and anchor condition at complete plate debonding: Slab S5 (- debonding crack, partially ruptured anchor, $\bullet$ undamaged anchor)

Figure 5. FRP-anchored FRP-strengthened slab test results.

\section{FRP-TO-METAL}

Decidedly less research activity has been directed towards the application of FRP to metal than FRP to concrete. A recent review by Zhao and Zhang (2007) highlighted limitations in our understanding of the interfacial behaviour between the FRP and its metal substrate. Recent efforts by the research community are, however, addressing the bond issue (e.g. Xia and Teng 2005, Akbar et al. 2010). At HKU, experimental investigations are being conducted on the bond strength and behaviour of FRP-to-metal joints. The main test parameters being systematically investigated, which have received little to no attention to date but which are considered of importance, are

- Surface preparation: mechanical (i.e. sanding, grinding, needling), and chemical (i.e. acid etching) treatments,

- Metal type: (i.e. non-galvanised mild steel, stainless steel, aluminium), and

- Geometry: bond length and identification of effective bond length.

Research efforts are ultimately directed towards the development of bond strength and bond stressslip models. A brief treatment of some of the surface preparation research, which is contained in more detail in Kim et al. (2010), is presented as follows in addition to some more recent work and a discussion on suitable analytical modeling approaches.

\subsection{FRP-to-metal joint tests}

The single-shear FRP-to-metal joint test set-up shown in Figure 6 has been utilised to date. In order to minimise bending of the test specimens, the metal plates were stiffened to produce a flexural rigidity ratio between the metal and FRP of 13,000. The setup is simple, quick to assemble, and easy to test.
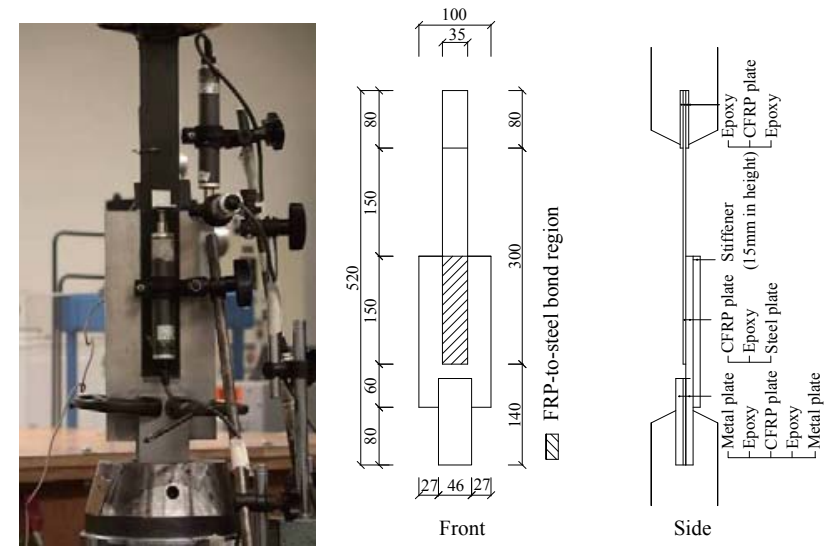

Figure 6. Stiffened single-shear FRP-to-metal joint test set-up.

Tests on different mechanical surface preparation techniques of sanding, grinding and needling on mild-steel and stainless steel has enabled identification of the following failure modes:

Mode 1: Adherend failure in oxidised layer, Mode 2A: Thin-layer cohesive failure in metal-toadhesive interface,

Mode 2B: Thin-layer cohesive failure in FRP-toadhesive interface, and

Mode 2C: Mixed thin-layer cohesive failure. 
Mode 1 (Figure 7a) was typically observed in unprepared mild-steel samples and if the oxidised layer was not sufficiently removed. The strength of the bond between the oxidised layer and the base metal was clearly the limiting factor and this revealed an important practical issue. In mild steel, the epoxy needed to be applied to the exposed base metal straight after preparation in order to halt/delay oxidisation. For specimens exhibiting cohesive failure (e.g. Figure $7 b$ ), the effect of surface preparation was less obvious. There, however, appeared to be a correlation between rougher topology of the metal surface and reduced bond strength.

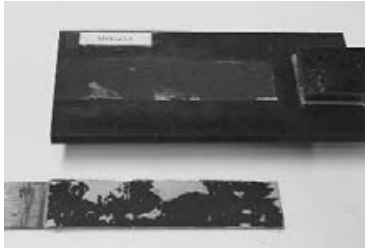

(a) Failure in oxidised layer

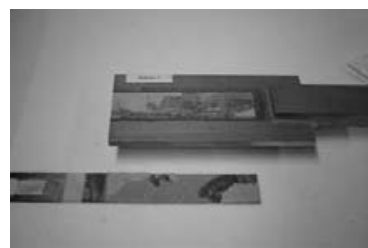

(b) Cohesive failure
Figure 7. FRP-to-metal joint failure modes.

Figure 8 shows slip and stress results obtained from strain gauge readings. Figure 8a shows a typical load-slip response for an FRP-to-stainless steel joint. The peak load plateau, corresponding to propagation of the debonding crack with little increase in load, is obvious. The corresponding strain versus distance at different levels of load is shown in Figure $8 \mathrm{~b}$ while the bond stress-distance and bond stress-slip relationships are shown in Figures 8c and $8 \mathrm{~d}$, respectively. The bi-linear nature of the bond stress-slip curves is evident and the low scatter of results is due to the homogeneity of the steel and the nature of the cohesive failure. This is in contrast the higher scattered FRP-to-concrete and FRP-to-timber joint results. Such scatter is attributed to debonding occurring in the non-homogenous adherends.

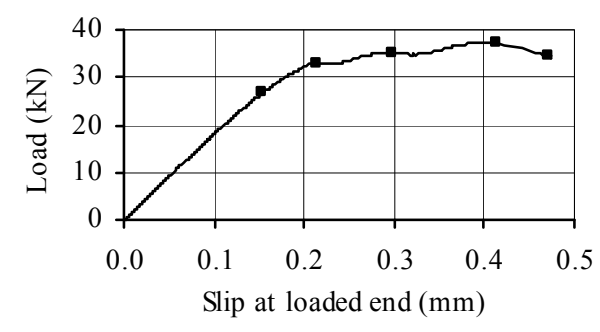

(a) Load-slip

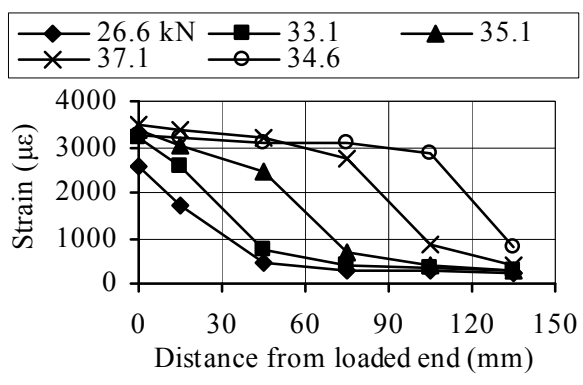

(b) Strain-distance

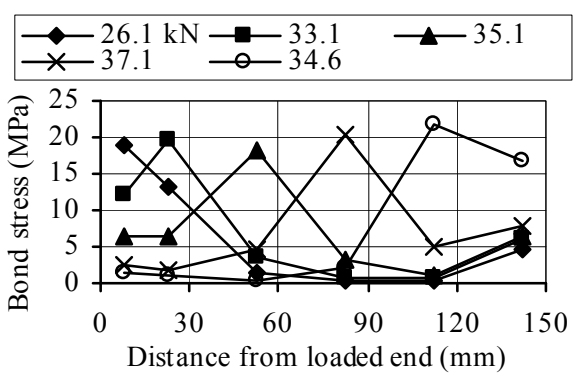

(c) Bond stress-distance

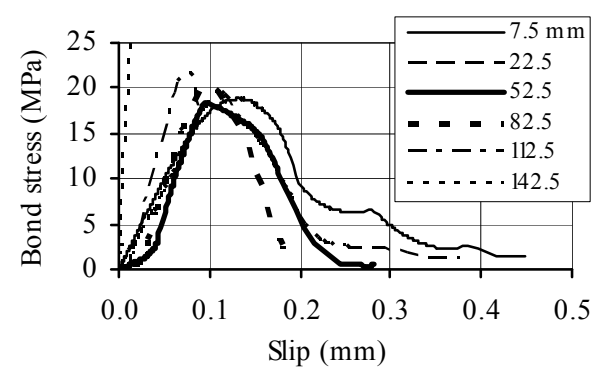

(d) Bond stress-slip

Figure 8. FRP-stainless steel joint (fine sanding) results.

Current work entails more testing and development of bond stress-slip models. Such models can be developed from bond-slip responses directly derived from strain gauges (e.g. Dai et al. 2003). Models can also be developed from measured load-slip responses (e.g. Dai et al. 2003, Zhou et al. 2010), the J-integral based approach, or other means (e.g. partial interaction approach of Akbar et al. 2010).

\section{FRP-TO-TIMBER}

Timber has been a commonly used construction material in civil infrastructure throughout the world for several millennia and still continues to be a popular construction material today. Externally bonded FRP composites offer strengthening and stiffening solutions to biologically degraded or mechanically overloaded timber members. In addition, the unobtrusiveness of FRP composites, particularly when used as near-surface mounted (NSM) reinforcement, offers tremendous potential for the repair and restoration of historical timber structures. The application of FRP to timber has received the least research activity to date, despite the popularity of timber.

The aim of the HKU research is to enhance understanding of the bonded FRP-to-concrete interface and to develop analytical models. The following is a summary of important aspects requiring investigation and understanding. Some of these issues are receiving attention to date already (e.g. Davalos et al. 2000, Raftery et al. 2009).

Timber: Species (hard/soft wood), grade, grain direction, density, moisture content (seasoned, unseasoned), pre-aging, weathering, natural growth characteristics (knots, checks, pith and core wood), 
existing cracks and damages, stress level (i.e. creep), treated and untreated wood, geometry.

FRP: Fibre type (carbon and glass), resin type (e.g. epoxy, polyester, phenolic), elastic modulus, geometry.

Adhesive: Type (e.g. epoxy, resorcinolformaldehyde (RF), vinylester), compatibility with wood and FRP, and type of primer (coupling agent), elastic modulus, geometry.

Loading direction and type: Parallel or perpendicular to grain, monotonic/cyclic.

Surface preparation: Sanding, planning, needling and wiping with solvents.

Durability: Effect of changing moisture content on FRP-to-timber bond.

\subsection{FRP-to-timber joint tests}

Tests on the bond strength and behaviour of FRP-totimber bonds are limited. The majority of tests have utilised a modified block-shear test of ASTM D90503 (2003). This test, which gives the shear strength of the bond as an average stress over the bonded plate area, has been used to date (e.g. Davalos et al. 2000, Raftery et al. 2009). As the FRP is sandwiched between two pieces of timber, its surface is difficult to monitor. In order to monitor the bonded interface and understand it in more detail, tests on single-shear FRP-to-timber joint specimens are being conducted at HKU (Figure 9).
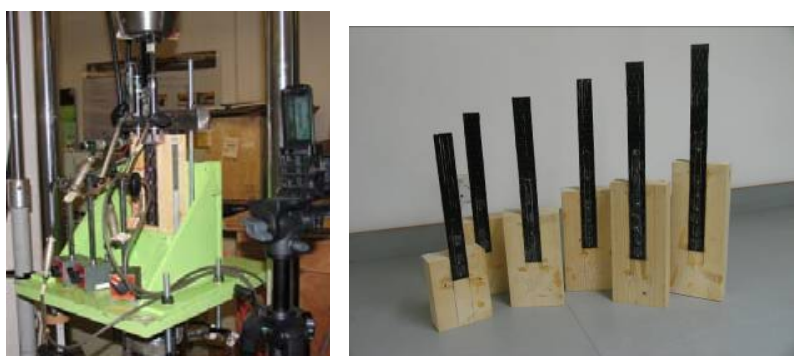

Figure 9. Single-shear FRP-to-timber joint tests.

To date, tests on seasoned pine (moisture content $\approx 10 \%$ ), which have been strengthened with externally bonded wet lay-up carbon fibre sheets, have been undertaken. The main test variables have been (i) length of bonded FRP, and (ii) natural growth characteristics of the timber (i.e. influence of direction of annual growth rings in relation to the bonded surface, and knots). Figure 10 provides a visual summary of many of the test specimens post-test. The failure planes in Figure 10 show that interfacial failure generally occurs in the timber (provided good quality FRP application exists). Such a failure is analogous to interfacial failure in the concrete in FRP-to-concrete joints. A more comprehensive account of these tests can be found in Wan et al. (2010), however, a brief over-view as well as more recent work and comments pertaining to analytical modeling are given as follows.

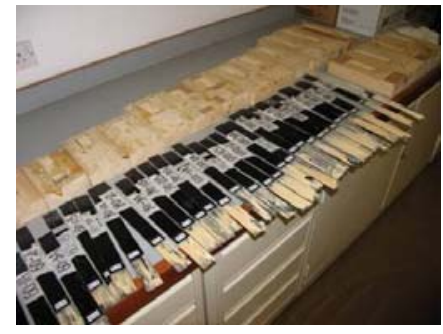

Figure 10. Debonded FRP plate and timber interfaces.

Figure 11 shows the relationship between strength and bonded FRP length for 52 joint tests. The effective bond length phenomenon exists in which the strength of the joint increases until a certain bond length is reached. Sides A and B are distinguished by the location of the harder pith wood in relation to the bonded FRP. This translates to FRP bonded to faces predominately perpendicular (Side A) or parallel (Side B) to the annual growth rings. Side B clearly provides a lower bound strength which is more governed by the interlaminar strength of the annual growth rings. The scatter of results amongst each bond length increases with increased length. Apart from inherent experimental variation, this scatter is also due to the presence of other natural growth characteristics in the timber such as knots. In light of the complexity and variability of timber, as revealed in Figure 11, much more testing is required.

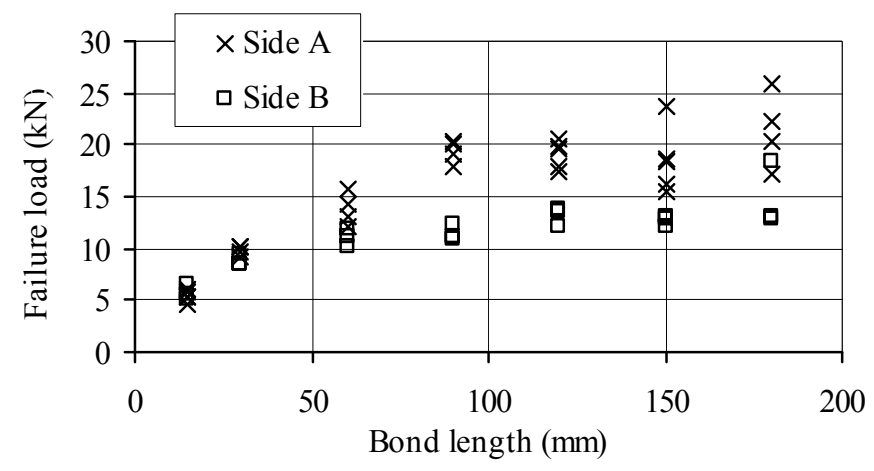

Figure 11. Failure load-bond length of FRP-to-timber joints.

The results of a typical joint of long bond length (i.e. $180 \mathrm{~mm}$ ) are shown in Figure 12 in which slips and stresses are obtained from strain gauge readings. Figure 12a shows a typical load-slip response and Figure $12 \mathrm{~b}$ the corresponding strain-distance response at selected levels of load. The flattening of the load-slip and strain-distance response indicates propagation of the debonding crack. The propagating debonding crack is also observed from the moving peak in the bond stress-distance responses in Figure 12c. The resulting bond stress-slip response at different positions along the FRP is then provided in Figure 12d. The familiar bi-linear bond stress-slip relation exists, however, the variability of results due to the highly variable nature of timber suggests bond-slip models would be better derived from the load-slip response (i.e. Dai et al. 2003, Zhou et al. 2010) or other methods (e.g. Akbar et al. 2010). 


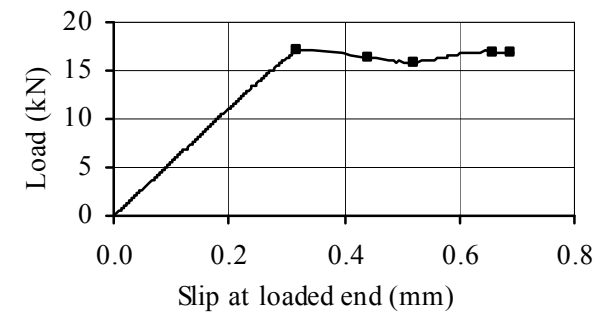

(a) Load-slip

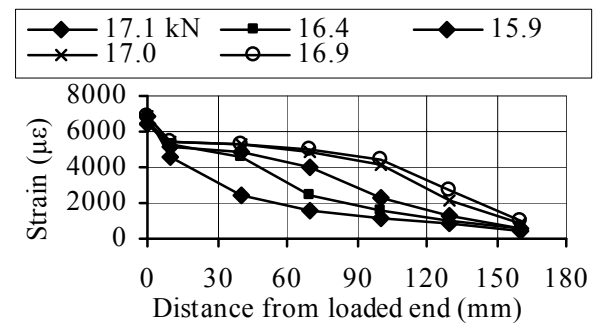

(b) Strain-distance

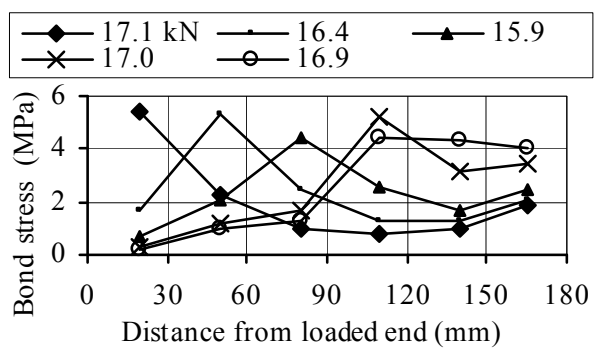

(c) Bond stress-distance

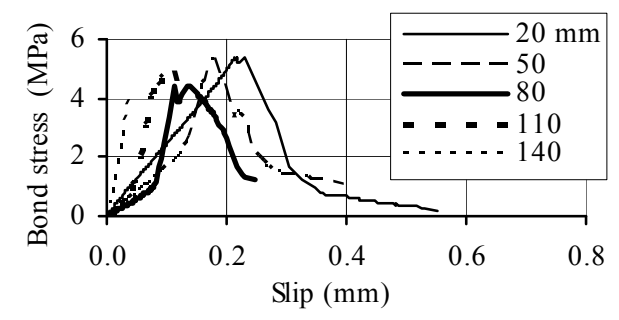

(d) Bond stress-slip

Figure 12. FRP-timber joint test results.

\section{DISCUSSION}

FRP-to-concrete and FRP-to-timber joints will generally experience interfacial failure in the concrete or timber adherends, respectively. Metal-to-FRP joints generally experience cohesive failure. The test results presented herein are, however, suitable for developing analytical models, via (i) strain gauge, (ii) load-slip, (iv) J-integral, and (v) partial interaction based approaches. It should be noted though that there are limitations in using strain gauge measurements for developing analytical models due to the discrete nature of the measurements.

\section{CONCLUSIONS}

Selected ongoing experimental research at The University of Hong Kong on the application of FRP to concrete, metal and timber materials has been presented herein. Some similarities and differences in behaviour between FRP bonded to concrete, metal and timber materials have been identified as well as potential analytical modeling approaches.

\section{ACKNOWLEDGEMENTS}

The financial assistance of the HK Research Grants Council (grants HKU 716308E, 715907E) and HKU (grants 10208970, 10400354) are acknowledged. The author also thanks his collaborators and graduate students, namely Profs P.Z. Qiao (WSU, USA) and B. Young (HKU); Drs R. Seracino (NCSU, USA) and S.J. Kim (HKU); Messers S.H. Hu, J.Q. Yang and H.W. Zhang; and Miss J. Wan (all HKU).

\section{REFERENCES}

ASTM D 905-03 2003. Strength Properties of Adhesive Bonds in Shear by Compression Loading, American Society for Testing and Materials (ASTM), PA, USA.

Akbar, I, Oehlers, DJ \& Mohamed Ali, MS 2010. Derivation of the bond-slip characteristics for FRP plates steel members, J. of Const. Steel Res., in-press.

Dai, JG, Ueda, T \& Sato, Y 2003. Development of the nonlinear bond stress-slip model of fiber reinforced plastics sheetconcrete interfaces with a simple model, J. Comp. for Const., ASCE, 9(1), 52-62.

Davalos JF, Qiao PZ \& Trimble BS 2000. Fiber-reinforced composite and wood bonded interfaces: Part 1. durability and shear strength", J. Comp. Tech. \& Res., 22(4), 224-231.

Hollaway, LC \& Teng, JG 2008. Strengthening and Rehabilitation of Civil Infrastructures using Fibre-reinforced Polymer (FRP) Composites, Woodhead Publishing, UK.

Kim, SJ, Smith, ST \& Young, B 2010. Effect of surface preparation on the strength of FRP-to mild steel and FRP-tostainless steel joints, Proc., Fifth Int. Conf. on FRP Comp. in Civil Eng., CICE 2010, Beijing, China.

Raftery, GM, Harte, AM \& Rodd, PD 2009. Bonding of FRP materials to wood using thin epoxy gluelines, Int. J. Adhesion \& Adhesives, 29, 580-588.

Smith, ST 2009. FRP anchors: recent advances in research and understanding, Proc., Second Asia-Pacific Conf. on FRP in Struct., APFIS 2009, Seoul, Korea, 35-44.

Smith, ST, Hu, SH, Kim, SJ \& Seracino, R 2010. FRPstrengthened RC slabs anchored with FRP anchors", Eng. Struct., under review.

Wan, J, Smith, ST \& Qiao, PZ 2010. FRP-to-softwood joints: experimental investigation", Proc., Fifth Int. Conf. on FRP Comp. in Civil Eng., CICE 2010, Beijing, China.

Xia, SH \& Teng, JG 2005. Behaviour of FRP-to-steel bonded joints, Proc., Int. Symp. on Bond Behaviour of FRP in Struct., BBFS 2005, Hong Kong, 411-418.

Zhang, HW \& Smith ST 2010. FRP-to-concrete joint assemblies anchored with multiple FRP anchors: experimental investigation, Proc., Fifth Int. Conf. on FRP Comp. in Civil Eng., CICE 2010, Beijing, China.

Zhao. XL \& Zhang, L 2007. State-of-the-art review on FRP strengthened steel structures, Eng. Struct., 29, 1808-1823.

Zhou, YW, Wu, YF \& Yun, YC 2010. Analytical modeling of the bond-slip relationship at FRP-to-concrete interfaces for adhesively bonded joints, Comp. Part B, in-press. 